\title{
金催化氧化末端炔烃合成 $\alpha$-乙酰氧基甲基酮方法研究
}

\author{
王小勇＼cjkstart李治章* \\ (湖南科技学院生命科学与化工系 永州 425100)
}

\begin{abstract}
摘要 采用 $\mathrm{Ph}_{3} \mathrm{PAuNTf}_{2}$ 作为催化剂, 8-甲基喹啉氮氧化物为氧化剂, 甲基磺酸为添加酸, 金催化氧化末端炔烃与乙酸 分子反应，室温下高效合成了一系列 $\alpha$-乙酰氧基甲基酮化合物. 考察了氧化剂、催化剂结构、催化剂用量等因素对反 应的影响, 确定了最优反应条件, 最高收率为 $90 \%$. 提出了 $\alpha$-羊炭基金卡宾对乙酸分子 $\mathrm{O}-\mathrm{H}$ 键的卡宾反应机理. 该方法 具有操作简单、条件温和、产率高等特点, 为含有 $\alpha$-乙酰氧基甲基酮结构的化合物合成提供了一条新的途径. 关键词 金催化; 炔烃; 分子间氧化; $\alpha$-羰基金卡宾中间体; $\alpha$-乙酰氧基甲基酮
\end{abstract}

\section{Gold(I)-Catalyzed Intermolecular Oxidation of Terminal Alkynes: Synthesis of $\alpha$-Acetoxy Ketones}

\author{
Wang, Xiaoyong Li, Zhizhang* \\ (Department of Life Science and Chemical Engineering, Hunan University of Science and Engineering, Yongzhou 425100)
}

\begin{abstract}
In the presence of $\mathrm{Ph}_{3} \mathrm{PAuNTf}_{2}, 8$-methylquinoline- $N$-oxide and $\mathrm{MsOH}$, various of terminal alkynes were efficiently converted into the corresponding $\alpha$-acetoxy ketones. The influences of oxidant, catalyst structure, catalyst loading and reaction media on the reaction were investigated, and the optimal reaction conditions were also obtained. The reaction is proposed to proceed via $\alpha$-oxo gold carbene intermolecular reaction with acetic acids. The reaction method with its advantages of simple procedure, mild reaction conditions and high yield provides a novel valuable approach to $\alpha$-acetoxy ketones from terminal alkynes.
\end{abstract}

Keywords gold catalysis; alkynes; intermolecular oxidation; $\alpha$-oxo gold carbene; $\alpha$-acetoxy ketones

近年来，均相金催化反应逐渐成为有机化学中的热 点领域之一. 金配合物的一个优点在于它具有较强的活 化碳碳多重键的能力, 使其能够在温和条件下接受多种 亲核试剂的进攻. 利用该活化机制, 化学家们发展了一 系列金催化碳、氧、硫、氮等亲核试剂对碳碳不饱和键 的亲核加成反应, 从而使简单不饱和烃底物通过该类反 应实现一系列多种键和官能团的有效转化, 为简便高效 合成结构复杂分子提供了新途径 ${ }^{[1 ~ 6]}$. 利用吡啶/喹啉氮

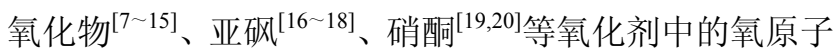
亲核进攻被金活化的炔基生成 $\alpha$-羰基金卡宾, 其与亲核 试剂发生串联反应，从而构建一系列的复杂分子，该方 法是金催化化学的一个重大进展 ${ }^{[21]}$.

$\alpha$-乙酰氧基甲基酮是重要的有机合成中间体, 广泛 用于有机化学及药物化学中 ${ }^{[22]}$. 其主要合成方法包括

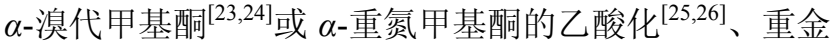
属氧化剂氧化酮或酮的衍生物等 ${ }^{[27 ~ 29]}$. 这些反应都有 着不可避免的缺陷，如反应产率低、反应条件苛刻、使 用或产生有毒化学物质. 本文研究一种具有绿色安全、 原子经济性高、实验操作及后处理简便等特点的炔基生 成 $\alpha$-羰基金卡宾及其串联反应的方法.

2012 年, Zhang 等 ${ }^{[12]}$ 报道了金催化氧化炔烃与二氯 乙烷的反应. 在这个反应中, 金催化分子间氧化炔烃生 成的 $\alpha$-羰基金卡宾中间体表现出非常高的亲电性(与铑 盐催化分解 $\alpha$-羰基重氮化合物生成的铑卡宾活性接近), 金卡宾捕获卤代溶剂的卤原子生成 $\alpha$-卤代甲基酮. 基于 该原理，本文利用 $\alpha$-羰基金卡宾捕获含氧溶剂的氧原 子, 如乙酸, 生成 $\alpha$-乙酰氧基甲基酮化合物, 并获得了 理想的实验结果.

\footnotetext{
*E-mail: 1zz5608@126.com

Received August 11, 2013; revised November 4, 2013; published online November 14, 2013. ince.

湖南省自然科学基金(No. 11JJ2009)和湖南省高校科技创新团队资助项目.
}

Project supported by the Natural Science Foundation of Hunan Province (No. 11JJ2009) and the Sci-Tech Innovation Teams in Universities of Hunan Prov- 


\section{1 结果与讨论}

\section{1 反应条件优化}

首先, 考察了辛炔与乙酸的反应. 辛炔 $(1.0 \mathrm{mmol})$, 8-甲基喹啉氮氧化物 $(1.3 \mathrm{mmol}$ ), 甲基磺酸 $(1.1 \mathrm{mmol}$ ), 5 mol $\% \mathrm{Ph}_{3} \mathrm{PAuNTf}_{2}, 1 \mathrm{~mL}$ 乙酸, 用 TLC 监控反应, 室温 搅拌反应 $6 \mathrm{~d}$ 后, 辛炔完全消失, 以 $76 \%$ 的产率获得 $\alpha$ 乙酰氧基甲基酮产物 $\mathbf{2 a}$, 同时还有 $8 \%$ 的甲基磺酸酯 (3a)副产物生成(表 1, Entry 2). 采用上述投料比, 以辛 炔与乙酸为模型, 考察了不同氧化剂对反应的影响. 在 一系列的氧化剂中, 8-甲基喹啉氮氧化物 $4 \mathrm{~b}$ 的氧化效果 最佳. 同时, 各种催化剂的篮选实验结果表明 $\mathrm{PhP}_{3} \mathrm{AuNTf}_{2}$ 催化效果最佳. 考虑到金催化剂的价格因 素, 探索了降低催化剂用量对反应的影响, 当催化剂用 量从 $5 \mathrm{~mol} \%$ 降低到 $4 \mathrm{~mol} \%$ 时, 产率基本没有变化(分离 收率 74\%). 但是降低催化剂用量至 $3 \mathrm{~mol} \%$ 时, 产率降 低至 62\%. 所以优化后的该反应条件选择： $4 \mathrm{~mol} \%$ $\mathrm{PhP}_{3} \mathrm{AuNTf}_{2}$ 为催化剂, 8-甲基喹啉氮氧化物 (1.3 mol)为 氧化剂, 甲基磺酸 $(1.1 \mathrm{~mol})$ 为添加酸, 室温摚拌反应.

\section{2 反应底物的扩充}

在最优化的反应条件下, 考察了不同取代末端炔烃 与乙酸的反应, 结果见表 2.
从表 2 中可知, 各种取代末端炔烃和乙酸都能顺利 地反应，以中等到高的收率得到产物. 具有卤素、甲基 醚、对甲基苯磺酸酯、乙酸酯的脂肪炔烃 $(2 \mathbf{b} \sim 2 \mathrm{e})$. 在 该反应中均不会影响. 大位阻的 3,3-二甲基丁炔、环丙 基乙炔都能顺利完成该反应，收率分别为 78\%和 $90 \%$ $(\mathbf{2 f}, 2 \mathrm{~g})$. 从芳炔与乙酸的反应可以看出, 芳环上取代基 的电子效应对反应的影响较小, 芳环上存在供电子取代 基(如 $\mathrm{Me}, i-\mathrm{Pr}$ 和 $\mathrm{MeO}$ )或者是吸电子取代基(如 $\mathrm{NO}_{2}, \mathrm{Cl}$ 和 I), 取代苯乙炔均能以优秀的产率获得相应的乙酰氧 基苯乙酮产物 $\mathbf{2 h} \sim \mathbf{2 0}$. 邻溴苯乙炔的邻位溴原子的位 阻作用对反应有一定的不利影响，但是仍然能以 $70 \%$ 的 产率得到相应的产物 2q.

\section{2 反应机理}

在前人研究 $\alpha$-羰基金卡宾分子间反应的基础

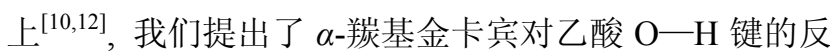
应(Scheme 1)。阳离子金催化剂活化炔基，8-甲基喹啉氮 氧化物的氧亲核进攻金活化的炔基生成 $\alpha$-羰基金卡宾 中间体 $\mathbf{A}$, 乙酸的分子与卡宾中间 $\mathbf{A}$ 发生加成反应生成 氧嗡中间体 $\mathbf{B}$, 中间体 $\mathbf{B}$ 发生质子去氢化生成 $\alpha$-乙酰氧 基甲基酮 2. 同时，金卡宾也会发生对甲基磺酸的 $\mathrm{O}-\mathrm{H}$ 键的插入反应生成副产物 $\alpha$-甲基磺酸酯甲基酮 $\mathbf{3}$, 由于 乙酸的浓度远远高于甲基磺酸, 所以产物 $\mathbf{2}$ 是主要产物.

表 1 反应条件的优化 ${ }^{a}$

Table 1 Reaction condition screening

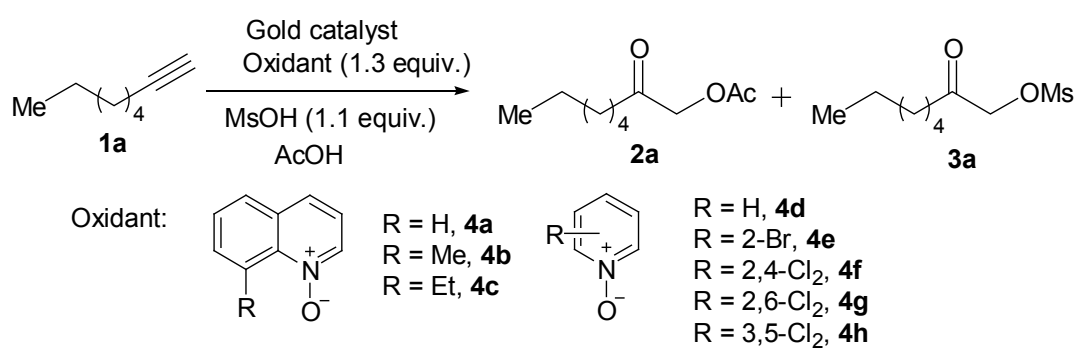

\begin{tabular}{|c|c|c|c|c|}
\hline Entry & Catalyst & Oxidant & Condition & Yield $^{b} / \%$ \\
\hline 1 & $5 \mathrm{~mol}^{2} \mathrm{Ph}_{3} \mathrm{PAuNTf}_{2}$ & $4 a$ & 8 h, r.t. & 58 \\
\hline 2 & $5 \mathrm{~mol}^{2} \mathrm{Ph}_{3} \mathrm{PAuNTf}_{2}$ & $4 b$ & 8 h, r.t. & 76 \\
\hline 3 & $5 \mathrm{~mol}^{2} \mathrm{Ph}_{3} \mathrm{PAuNTf}_{2}$ & $4 c$ & 10 h, r.t. & 70 \\
\hline 4 & $5 \mathrm{~mol}^{2} \mathrm{Ph}_{3} \mathrm{PAuNTf}_{2}$ & 4d & 8 h, r.t. & 33 \\
\hline 5 & $5 \mathrm{~mol}^{2} \mathrm{Ph}_{3} \mathrm{PAuNTf}_{2}$ & $4 e$ & 8 h, r.t. & 35 \\
\hline 6 & $5 \mathrm{~mol}^{2} \mathrm{Ph}_{3} \mathrm{PAuNTf}_{2}$ & $4 f$ & 8 h, r.t. & 20 \\
\hline 7 & $5 \mathrm{~mol} \% \mathrm{Ph}_{3} \mathrm{PAuNTf}_{2}$ & $4 g$ & 8 h, r.t. & 46 \\
\hline 8 & $5 \mathrm{~mol} \% \mathrm{Ph}_{3} \mathrm{PAuNTf}_{2}$ & $4 h$ & 8 h, r.t. & 48 \\
\hline 9 & 5 mol\% Cy-JohnPhosAuNTf 2 & $4 b$ & 12 h, r.t. & 52 \\
\hline 10 & 5 mol\% MePhosAuNTf ${ }_{2}$ & $4 b$ & 8 h, r.t. & 50 \\
\hline 11 & $5 \mathrm{~mol}^{2} \mathrm{Et}_{3} \mathrm{PAuNTf}_{2}$ & $4 b$ & 8 h, r.t. & 41 \\
\hline 12 & $5 \mathrm{~mol}^{2} \mathrm{IPrAuNTf}_{2}$ & $4 b$ & 8 h, r.t. & 29 \\
\hline 13 & $4 \mathrm{~mol} \% \mathrm{Ph}_{3} \mathrm{PAuNTf}_{2}$ & $4 b$ & 8 h, r.t. & $74^{b}$ \\
\hline 14 & $3 \mathrm{~mol} \% \mathrm{Ph}_{3} \mathrm{PAuNTf}_{2}$ & $4 b$ & $8 \mathrm{~h}, \mathrm{rt}$ & 62 \\
\hline
\end{tabular}

$\bar{a}$ The yield was determined by ${ }^{1} \mathrm{H}$ NMR spectroscopy using mesitylene as an internal reference. ${ }^{b}$ Isolated yields after silica gel column chromatography. 
表 2 合成 $\alpha$-乙酰氧基甲基酮 ${ }^{a}$

Table 2 Synthesis of $\alpha$-acetoxy ketones

$$
\mathrm{R} \underset{1}{\frac{4 \mathrm{~mol} \% \mathrm{Ph}_{3} \mathrm{PAuNTf}_{2}, \mathbf{4 b} \text { (1.3 equiv.) }}{\mathrm{MsOH}(1.1 \text { equiv.), } \mathrm{AcOH} \text {, r.t., } 8 \mathrm{~h}}} \mathrm{R} \overbrace{2}^{\mathrm{O} O A c}
$$

\begin{tabular}{|c|c|c|c|c|c|c|c|}
\hline Entry & Product & 2 & Yield/\% & Entry & Product & 2 & Yield/\% \\
\hline 1 & & $2 b$ & 62 & 2 & & $2 \mathrm{c}$ & 70 \\
\hline 3 & & 2d & 78 & 4 & & $2 e$ & 70 \\
\hline 5 & & $2 f$ & 78 & 6 & & $2 \mathrm{~g}$ & 90 \\
\hline 7 & & $2 \mathrm{~h}$ & 83 & 8 & & $2 \mathbf{i}$ & 81 \\
\hline 9 & & $2 j$ & 82 & 10 & & $2 \mathrm{k}$ & 75 \\
\hline 11 & & 21 & 70 & 12 & & $2 m$ & 77 \\
\hline 13 & & $2 n$ & 84 & 14 & & 20 & 82 \\
\hline 15 & & $2 p$ & 73 & 16 & & $2 q$ & 60 \\
\hline
\end{tabular}

${ }^{a}$ Reaction conditions: alkyne $(0.3 \mathrm{mmol}), 8$-methylquinoline- $N$-oxide $(0.39 \mathrm{mmol})$, catalyst $(5 \mathrm{~mol} \%)$, AcOH $(3 \mathrm{~mL})$, monitored by TLC, r.t. ${ }^{b}$ Isolated yields after silica gel column chromatography.

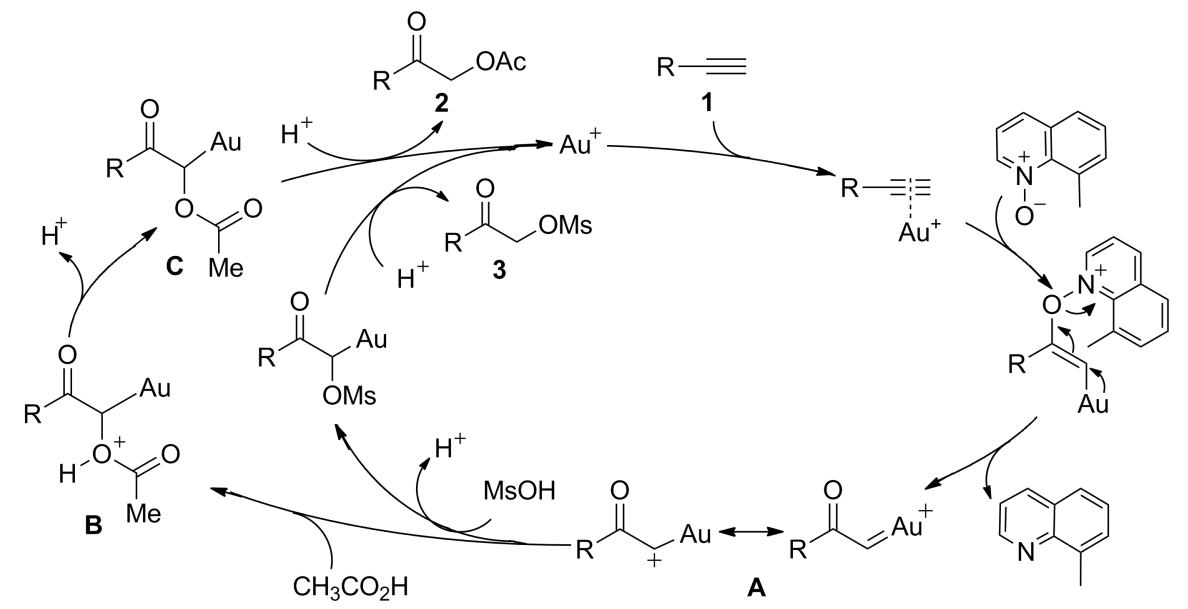

Scheme 1 


\section{2 结论}

发展了一种简洁高效、绿色的 $\alpha$-乙酰氧基甲基酮的 合成方法. 研究结果表明: 在 $\mathrm{PhP}_{3} \mathrm{AuNTf}_{2}$ (4 mol\%), 8甲基喹啉氮氧化物 $(1.3 \mathrm{mmol})$, 甲基磺酸 $(1.1 \mathrm{mmol})$, 乙 酸 $(1 \mathrm{~mL})$ 的反应条件下, 各种末端炔烃与乙酸能够得到 中等及优秀的产率. 反应机理包括均相金催化分子间氧 化末端炔烃生成 $\alpha$-羰基金卡宾中间体，及金卡宾对乙酸 分子 $\mathrm{O}-\mathrm{H}$ 健的分子间插入反应. 该反应原料得, 底物 适用性广, 应条件温和, 实验操作简单和产物易分离等 特点. $\alpha$-羰基金卡宾中间体对固体羧酸、磺酸等酸类化 合物 $\mathrm{O}-\mathrm{H}$ 的插入反应正在研究中, 将在之后的论文中 报道.

\section{3 实验部分}

\section{1 试剂与仪器}

${ }^{1} \mathrm{H} \mathrm{NMR}$ (以 $\mathrm{TMS}$ 为内标) 和 ${ }^{13} \mathrm{C} \mathrm{NMR}$ (以 $\mathrm{TMS}$ 为内 标)使用 Varian INOVA-400 型核磁共振仪测定; 红外光 谱用 Perkin Elmer FT-IR spectrum 2000 spectrometer 测 定 (波数单位是 $\mathrm{cm}^{-1}$ ); 质谱用 Thermo-Finnigan LCQ-Advantage 型质谱仪(美国)测定, 测定模式为 Ion source: APCI(大气压化学电离, 正离子模式). 所有药品 和试剂均为分析纯, 购自百灵威试剂公司.

\section{2 目标产物的合成}

室温下, 在 $10 \mathrm{~mL}$ 反应管中, 依次加入 $3 \mathrm{~mL}$ 乙酸, 末端炔烃 $(0.3 \mathrm{mmol})$, 氮氧化物 $4 \mathrm{~b}$ (0.39 mmol, 62.01 $\mathrm{mg}), \mathrm{MsOH}$ (0.33 mmol, $31.71 \mathrm{mg}), \mathrm{Ph}_{3} \mathrm{PAuNTf}_{2}(0.015$ $\mathrm{mmol}, 11.1 \mathrm{mg})$, 室温搅拌, 用 TLC 监测反应进程, 一 般摚拌 $8 \mathrm{~d}$. 反应完毕, 减压旋去溶剂, 剩余油状物或固 体使用适当比例的正己烷, 乙酸乙酯淋洗液通过柱层析 色谱进行分离得到纯产物 $\mathbf{2 a} \sim \mathbf{2 u}$.

2-羰基乙酸己酯 $(2 \mathbf{a})^{[30]}$ : 白色固体，产率 74\%. m.p. $63 \sim 66{ }^{\circ} \mathrm{C} ;{ }^{1} \mathrm{H}$ NMR $\left(400 \mathrm{MHz}, \mathrm{CDCl}_{3}\right) \delta: 0.88(\mathrm{t}, J=6.8$ $\mathrm{Hz}, 3 \mathrm{H}), 1.28$ (brs, 6H), 1.62 (t, $J=7.2 \mathrm{~Hz}, 2 \mathrm{H}), 2.16$ (s, $3 \mathrm{H}), 2.40(\mathrm{t}, J=7.2 \mathrm{~Hz}, 2 \mathrm{H}), 4.66(\mathrm{~s}, 2 \mathrm{H}) ;{ }^{13} \mathrm{C}$ NMR $(100$ $\left.\mathrm{MHz}, \mathrm{CDCl}_{3}\right) \delta: 14.10,20.36,22.35,23.12,28.70,31.44$, 38.70, 67.89, 170.21, 204.02; IR (neat) v: 2970, 1753, 1733, 1365, 1221, $1052 \mathrm{~cm}^{-1}$; HRMS (EI) calcd for $\mathrm{C}_{14} \mathrm{H}_{26} \mathrm{O}_{3}$ : 186.1232; found 186.1236 .

2-羰基-6-氯乙酸己脂(2b): 黄色油状物, 产率 $62 \%$. ${ }^{1} \mathrm{H}$ NMR (400 MHz, $\left.\mathrm{CDCl}_{3}\right) \delta: 1.77 \sim 1.80(\mathrm{~m}, 4 \mathrm{H}), 2.16$ $(\mathrm{s}, 3 \mathrm{H}), 2.39(\mathrm{t}, J=6.8 \mathrm{~Hz}, 2 \mathrm{H}), 3.55(\mathrm{t}, J=6.0 \mathrm{~Hz}, 2 \mathrm{H})$, $4.66(\mathrm{~s}, 2 \mathrm{H}) ;{ }^{13} \mathrm{C} \mathrm{NMR}\left(100 \mathrm{MHz}, \mathrm{CDCl}_{3}\right) \delta: 20.51,31.74$, $37.85,44.52,67.93,170.38,203.37$; IR (neat) v: 3210, $2325,1735,1405,1240,1068,675 \mathrm{~cm}^{-1}$. HRMS (EI) calcd for $\mathrm{C}_{8} \mathrm{H}_{13} \mathrm{ClO}_{3}$ : 192.0553; found 192.0555 .

6-甲氧基-2-羰基乙酸己脂(2c): 白色油状物, 产率 $70 \%$. ${ }^{1} \mathrm{H}$ NMR (400 MHz, $\left.\mathrm{CDCl}_{3}\right) \delta: 1.54 \sim 1.60(\mathrm{~m}, 2 \mathrm{H})$, $1.62 \sim 1.69(\mathrm{~m}, 2 \mathrm{H}), 2.15(\mathrm{~s}, 3 \mathrm{H}), 2.43(\mathrm{t}, J=7.2 \mathrm{~Hz}, 2 \mathrm{H})$, $3.30(\mathrm{~s}, 3 \mathrm{H}), 3.34(\mathrm{t}, J=6.0 \mathrm{~Hz}, 2 \mathrm{H}), 4.66(\mathrm{~s}, 2 \mathrm{H}) ;{ }^{13} \mathrm{C}$ NMR (100 MHz, $\left.\mathrm{CDCl}_{3}\right) \delta: 20.13,20.64,28.97,38.45$, 58.66, 67.83, 72.02, 170.55, 203.82; IR (neat) $v: 2910$, 2855, 1755, 1720, 1440, $990 \mathrm{~cm}^{-1}$. HRMS (EI) calcd for $\mathrm{C}_{9} \mathrm{H}_{16} \mathrm{O}_{4}$ : 188.1049; found 188.1046.

2-羰基-6-对甲苯磺酰基乙酸己脂(2d)：黄色固体， 产率 78\%. m.p. $101 \sim 104{ }^{\circ} \mathrm{C} ;{ }^{1} \mathrm{H}$ NMR $(400 \mathrm{MHz}$, $\left.\mathrm{CDCl}_{3}\right) \delta: 1.64 \sim 1.66(\mathrm{~m}, 4 \mathrm{H}), 2.14(\mathrm{~s}, 3 \mathrm{H}), 2.40(\mathrm{t}, J=6.8$ $\mathrm{Hz}, 2 \mathrm{H}), 2.46$ (s, 3H), $4.00(\mathrm{t}, J=6.0 \mathrm{~Hz}, 2 \mathrm{H}), 4.60(\mathrm{~s}, 2 \mathrm{H})$, 7.32 (d, $J=8.2 \mathrm{~Hz}, 2 \mathrm{H}), 7.75$ (d, $J=8.2 \mathrm{~Hz}, 2 \mathrm{H}) ;{ }^{13} \mathrm{C}$ NMR $\left(100 \mathrm{MHz}, \mathrm{CDCl}_{3}\right) \delta: 19.01,20.41,21.66,28.10$, 37.63, 67.89, 70.04, 127.85, 129.78, 132.84, 144.81, 170.25, 203.28; IR (neat) $v: 2940,1740,1724,1590,1340$, 1086, $820 \mathrm{~cm}^{-1}$. HRMS (EI) calcd for $\mathrm{C}_{15} \mathrm{H}_{20} \mathrm{O}_{6} \mathrm{~S}$ : 328.0981 ; found 328.0984 .

1,6-二乙酰氧基-2-己酮(2e): 黄色固体，产率 70\%. m.p. $62 \sim 65{ }^{\circ} \mathrm{C} ;{ }^{1} \mathrm{H}$ NMR $\left(400 \mathrm{MHz}, \mathrm{CDCl}_{3}\right) \delta: 1.63 \sim$ $1.65(\mathrm{~m}, 4 \mathrm{H}), 2.02(\mathrm{~s}, 3 \mathrm{H}), 2.15(\mathrm{~s}, 3 \mathrm{H}), 2.43(\mathrm{t}, J=6.8 \mathrm{~Hz}$, $2 \mathrm{H}), 4.01(\mathrm{t}, J=6.0 \mathrm{~Hz}, 2 \mathrm{H}), 4.62(\mathrm{~s}, 2 \mathrm{H}) ;{ }^{13} \mathrm{C}$ NMR $(100$ $\left.\mathrm{MHz}, \mathrm{CDCl}_{3}\right) \delta: 19.45,20.33,20.78,27.83,37.99,63.87$, 67.81, 170.20, 171.05, 203.41; IR (neat) $v$ : 2959, 1741, 1729, 1370, 1218, 1055, $914 \mathrm{~cm}^{-1}$. HRMS (EI) calcd for $\mathrm{C}_{10} \mathrm{H}_{16} \mathrm{O}_{5}$ : 216.0998; found 216.0995.

3,3 二甲基-2-羰基乙酸丁酯(2f) ${ }^{[31]}$ : 黄色油状液体, 产率 78\%. ${ }^{1} \mathrm{H}$ NMR (400 $\left.\mathrm{MHz}, \mathrm{CDCl}_{3}\right) \delta: 1.18(\mathrm{~s}, 9 \mathrm{H})$, $2.14(\mathrm{~s}, 3 \mathrm{H}), 4.85(\mathrm{~s}, 2 \mathrm{H}) ;{ }^{13} \mathrm{C} \mathrm{NMR}\left(100 \mathrm{MHz}, \mathrm{CDCl}_{3}\right) \delta$ : $20.45,26.15,42.67,64.40,170.33,207.89$; IR (neat) $v$ : 2930, 1755, 1726, 1356, $1240 \mathrm{~cm}^{-1}$. HRMS (EI) calcd for $\mathrm{C}_{8} \mathrm{H}_{14} \mathrm{O}_{3}$ : 158.0943; found 158.0938.

2-环丙基-2-羰基乙酸乙酯 $(\mathbf{2 g})^{[32]}$ : 黄色油状液体, 产率 $78 \%$. ${ }^{1} \mathrm{H}$ NMR $\left(400 \mathrm{MHz}, \mathrm{CDCl}_{3}\right) \delta: 0.93 \sim 0.97(\mathrm{~m}$, $2 \mathrm{H}), 1.07 \sim 1.12(\mathrm{~m}, 2 \mathrm{H}), 1.92 \sim 1.96(\mathrm{~m}, 1 \mathrm{H}), 2.15(\mathrm{~s}$, $3 \mathrm{H}), 4.80(\mathrm{~s}, 2 \mathrm{H}) ;{ }^{13} \mathrm{C}$ NMR (100 MHz, $\left.\mathrm{CDCl}_{3}\right) \delta: 11.34$, $17.11,20.46,68.35,170.08,203.65$; IR (neat) $v: 2995$, 2935, 1738, 1705, 1235, $898 \mathrm{~cm}^{-1}$. HRMS (EI) calcd for $\mathrm{C}_{7} \mathrm{H}_{10} \mathrm{O}_{3}$ : 142.0630; found 142.0625 .

2-乙酰氧基苯乙酮 $(\mathbf{2 h})^{[31]}$ : 黄色油状液体, 产率 83\%. ${ }^{1} \mathrm{H}$ NMR (400 MHz, $\mathrm{CDCl}_{3}$ ) $\delta: 2.23$ (s, 3H), 5.35 (s, 2H), 7.48 (t, $J=8.0 \mathrm{~Hz}, 2 \mathrm{H}), 7.61(\mathrm{t}, J=7.6 \mathrm{~Hz}, 1 \mathrm{H}), 7.90$ $(\mathrm{d}, J=7.2 \mathrm{~Hz}, 2 \mathrm{H}) ;{ }^{13} \mathrm{C} \mathrm{NMR}\left(100 \mathrm{MHz}, \mathrm{CDCl}_{3}\right) \delta: 20.61$, $66.05,127.67,128.68,133.90,134.01,170.24,192.22$; IR 
(neat) $v: 2920,1750,1705,1580,1386,1230,956 \mathrm{~cm}^{-1}$. HRMS (EI) calcd for $\mathrm{C}_{10} \mathrm{H}_{10} \mathrm{O}_{3}: 178.0630$; found 178.0626.

2-(4-甲基苯基)-2-羰基乙酸乙酯(2i) ${ }^{[31]}$ : 白色固体, 产率 81\%. m.p. 82 $85{ }^{\circ} \mathrm{C} ;{ }^{1} \mathrm{H}$ NMR $\left(400 \mathrm{MHz}, \mathrm{CDCl}_{3}\right)$ $\delta: 2.22(\mathrm{~s}, 3 \mathrm{H}), 2.42(\mathrm{~s}, 3 \mathrm{H}), 5.31(\mathrm{~s}, 2 \mathrm{H}), 7.28$ (d, $J=8.0$ $\mathrm{Hz}, 2 \mathrm{H}), 7.82(\mathrm{~d}, J=8.0 \mathrm{~Hz}, 2 \mathrm{H}) ;{ }^{13} \mathrm{C}$ NMR $(100 \mathrm{MHz}$, $\left.\mathrm{CDCl}_{3}\right) \delta: 20.63,21.72,65.90,127.83,129.56,131.59$, 144.84, 170.45, 191.67; IR (neat) v: 2960, 1754, 1701, 1348, 1171, $962 \mathrm{~cm}^{-1}$. HRMS (EI) calcd for $\mathrm{C}_{11} \mathrm{H}_{12} \mathrm{O}_{3}$ : 192. 0786; found 192.0783.

2-(4-叔丁基苯基)-2-羰基乙酸乙酯(2j $)^{[33]}$ : 白色固 体, 产率 82\%. m.p. 91 96 ${ }^{\circ} \mathrm{C} ;{ }^{1} \mathrm{H}$ NMR $(400 \mathrm{MHz}$, $\left.\mathrm{CDCl}_{3}\right) \delta: 1.32(\mathrm{~s}, 9 \mathrm{H}), 2.20(\mathrm{~s}, 3 \mathrm{H}), 5.33(\mathrm{~s}, 2 \mathrm{H}), 7.50(\mathrm{~d}$, $J=8.4 \mathrm{~Hz}, 2 \mathrm{H}), 7.86(\mathrm{~d}, J=8.4 \mathrm{~Hz}, 2 \mathrm{H}) ;{ }^{13} \mathrm{C}$ NMR $(100$ $\left.\mathrm{MHz}, \mathrm{CDCl}_{3}\right) \delta: 20.46,30.82,35.06,65.85,125.58$, 127.45, 131.52, 157.60, 191.66; IR (neat) $v: 2970,1762$, 1692, 1085, $980 \mathrm{~cm}^{-1}$. HRMS (EI) calcd for $\mathrm{C}_{14} \mathrm{H}_{18} \mathrm{O}_{3}$ : 235.1334; found 235.1338.

2-(4-甲氧基苯基)-2-羰基乙酸乙酯(2k) ${ }^{[34]}$ : 白色固 体, 产率 75\%. m.p. $60 \sim 64{ }^{\circ} \mathrm{C} ;{ }^{1} \mathrm{H}$ NMR $(400 \mathrm{MHz}$, $\left.\mathrm{CDCl}_{3}\right) \delta: 2.22(\mathrm{~s}, 3 \mathrm{H}), 3.87(\mathrm{~s}, 3 \mathrm{H}), 5.29(\mathrm{~s}, 2 \mathrm{H}), 7.89(\mathrm{~d}$, $J=8.8 \mathrm{~Hz}, 2 \mathrm{H}), 7.95(\mathrm{~d}, J=8.8 \mathrm{~Hz}, 2 \mathrm{H}) ;{ }^{13} \mathrm{C}$ NMR $(100$ $\left.\mathrm{MHz}, \mathrm{CDCl}_{3}\right) \delta: 20.56,55.45,65.67,114.01,127.10$, 130.00, 164.03, 170.42, 190.56; IR (neat) v: 2940, 2857, 1754, 1704, 1349, 1161, $963 \mathrm{~cm}^{-1}$. HRMS (EI) calcd for $\mathrm{C}_{11} \mathrm{H}_{12} \mathrm{O}_{4}$ : 208.0736; found 208.0732.

2-(4-硝基苯基)-2-羰基乙酸乙酯(2I) ${ }^{[31]}$ : 白色固体, 产率 70\%. m.p. $120 \sim 124{ }^{\circ} \mathrm{C} ;{ }^{1} \mathrm{H}$ NMR $(400 \mathrm{MHz}$, $\left.\mathrm{CDCl}_{3}\right) \delta: 2.22(\mathrm{~s}, 3 \mathrm{H}), 5.33(\mathrm{~s}, 2 \mathrm{H}), 8.12(\mathrm{~d}, J=8.2 \mathrm{~Hz}$, $2 \mathrm{H}), 8.36(\mathrm{~d}, J=8.2 \mathrm{~Hz}, 2 \mathrm{H}) ;{ }^{13} \mathrm{C}$ NMR $\left(100 \mathrm{MHz}, \mathrm{CDCl}_{3}\right)$ $\delta: 20.36,66.12,124.05,128.85,138.52,150.58,170.30$, 191.06; IR (neat) $v: 2956,1750,1699,1489,1295,948$ $\mathrm{cm}^{-1}$. HRMS (EI) calcd for $\mathrm{C}_{10} \mathrm{H}_{9} \mathrm{NO}_{5}$ : 223.0534; found 223.0539 .

2-(4-氯苯基)-2-羰基乙酸乙酯 $(\mathbf{2 m})^{[31]}$ : 白色固体, 产率 77\%. m.p. 71 74 ${ }^{\circ} \mathrm{C} ;{ }^{1} \mathrm{H}$ NMR $\left(400 \mathrm{MHz}, \mathrm{CDCl}_{3}\right)$ $\delta: 2.22$ (s, 3H), 5.23 (s, 2H), 7.37 (d, $J=8.6 \mathrm{~Hz}, 2 \mathrm{H}), 7.79$ $(\mathrm{d}, J=8.6 \mathrm{~Hz}, 2 \mathrm{H}) ;{ }^{13} \mathrm{C}$ NMR $\left(100 \mathrm{MHz}, \mathrm{CDCl}_{3}\right) \delta: 20.40$, 65.82, 129.11, 129.25, 132.36, 140.48, 170.31, 191.05; IR (neat) $v: 2955,1750,1678,1512,1301,975 \mathrm{~cm}^{-1}$. HRMS (EI) calcd for $\mathrm{C}_{10} \mathrm{H}_{9} \mathrm{ClO}_{3}$ : 212.0224; found 212.0227.

2-(4-碘苯基)-2-羰基乙酸乙酯 $(\mathbf{2 n})^{[31]}$ : 白色固体, 产率 84\%. m.p. $110 \sim 114{ }^{\circ} \mathrm{C} ;{ }^{1} \mathrm{H}$ NMR $(400 \mathrm{MHz}$, $\left.\mathrm{CDCl}_{3}\right) \delta: 2.23$ (s, 3H), 5.30 (s, 2H), 7.61 (d, $J=7.8 \mathrm{~Hz}$,
$2 \mathrm{H}), 7.82(\mathrm{~d}, J=7.8 \mathrm{~Hz}, 2 \mathrm{H}) ;{ }^{13} \mathrm{C} \mathrm{NMR}\left(100 \mathrm{MHz}, \mathrm{CDCl}_{3}\right)$ $\delta: 20.46,65.32,101.85,128.91,134.45,138.28,170.40$, 191.56; IR (neat) v: 2960, 1751, 1699, 15381, 1319, 956 $\mathrm{cm}^{-1}$. HRMS (EI) calcd for $\mathrm{C}_{10} \mathrm{H}_{9} \mathrm{IO}_{3}: 303.9624$; found 303.9629 .

2-(4-苯基苯基)-2-羰基乙酸乙酯(20 $)^{[31]}$ : 白色固体, 产率 $82 \%$. m.p. $107 \sim 110{ }^{\circ} \mathrm{C} ;{ }^{1} \mathrm{H}$ NMR $(400 \mathrm{MHz}$, $\left.\mathrm{CDCl}_{3}\right) \delta: 2.24(\mathrm{~s}, 3 \mathrm{H}), 5.35(\mathrm{~s}, 2 \mathrm{H}), 7.48(\mathrm{~m}, 3 \mathrm{H}), 7.60(\mathrm{~d}$, $J=7.0 \mathrm{~Hz}, 2 \mathrm{H}), 7.72(\mathrm{~d}, J=7.4 \mathrm{~Hz}, 2 \mathrm{H}), 7.98$ (d, $J=7.4$ $\mathrm{Hz}, 2 \mathrm{H}) ;{ }^{13} \mathrm{C}$ NMR $\left(100 \mathrm{MHz}, \mathrm{CDCl}_{3}\right) \delta: 20.56,65.92$, $127.35,125.51,128.42,128.50,132.88,139.79,146.56$, 170.40, 191.66; IR (neat) $v: 2955,1711,1698,1310,920$, $760 \mathrm{~cm}^{-1}$. HRMS (EI) calcd for $\mathrm{C}_{16} \mathrm{H}_{14} \mathrm{O}_{3}$ : 254.0932; found 254.0936 .

2-(2-甲基苯基)-2-羰基乙酸乙酯(2p) ${ }^{[30]}$ : 白色油状 液体, 产率 $68 \% .{ }^{1} \mathrm{H}$ NMR $\left(400 \mathrm{MHz}, \mathrm{CDCl}_{3}\right) \delta: 2.20(\mathrm{~s}$, $3 \mathrm{H}), 2.52(\mathrm{~s}, 3 \mathrm{H}), 5.15(\mathrm{~s}, 2 \mathrm{H}), 7.24 \sim 7.28(\mathrm{~m}, 2 \mathrm{H}), 7.39 \sim$ $7.44(\mathrm{~m}, 1 \mathrm{H}), 7.60(\mathrm{~d}, J=7.2 \mathrm{~Hz}, 1 \mathrm{H}) ;{ }^{13} \mathrm{C}$ NMR $(100$ $\left.\mathrm{MHz}, \mathrm{CDCl}_{3}\right) \delta: 20.37,20.99,67.20,125.71,127.85$, $132.09,132.22,134.51,138.92,171.01,195.58$; IR (neat) $v: 2965,1742,1710,1348,1041,940 \mathrm{~cm}^{-1}$. HRMS (EI) calcd for $\mathrm{C}_{11} \mathrm{H}_{12} \mathrm{O}_{3}$ : 192.0786; found 192.0781 .

2-(2-澳苯基)-2-羰基乙酸乙酯(2q $)^{[31]}$ : 白色油状液 体, 产率 60\%. ${ }^{1} \mathrm{H}$ NMR $\left(400 \mathrm{MHz}, \mathrm{CDCl}_{3}\right) \delta: 2.16(\mathrm{~s}$, $3 \mathrm{H}), 5.15(\mathrm{~s}, 2 \mathrm{H}), 7.32 \sim 7.43(\mathrm{~m}, 2 \mathrm{H}), 7.49 \sim 7.52(\mathrm{~m}$, $1 \mathrm{H}), 7.62 \sim 7.65(\mathrm{~m}, 1 \mathrm{H}) ;{ }^{13} \mathrm{C}$ NMR $\left(100 \mathrm{MHz}, \mathrm{CDCl}_{3}\right) \delta$ : $20.41,67.67,119.32,127.45,129.54,132.53,133.78$, 138.22, 170.33, 196.54; IR (neat) v: 2980, 2942, 1724, 1683, 1601, 1215, $986 \mathrm{~cm}^{-1}$. HRMS (EI) calcd for $\mathrm{C}_{10} \mathrm{H}_{9} \mathrm{BrO}_{3}$ : 255.9735; found 255.9739.

\section{References}

[1] Fürstner, A.; Davies, P. W. Angew. Chem., Int. Ed. 2007, 46, 3410.

[2] Hashmi, A. S. K. Chem. Rev. 2007, 107, 3180.

[3] Gorin, D. J.; Sherry, B. D.; Toste, F. D. Chem. Rev. 2008, 108, 3351.

[4] Li, Z.; Brouwer, C.; He, C. Chem. Rev. 2008, 108, 3239.

[5] Huang, H.; Zhou, Y.; Liu, H. Beilstein J. Org. Chem. 2011, 7, 897.

[6] Lu, B.-L.; Dai, L.; Shi, M. Chem. Soc. Rev. 2012, 41, 3318.

[7] Ye, L.; He, W.; Zhang, L. J. Am. Chem. Soc. 2010, 132, 8550.

[8] Ye, L.; He, W.; Zhang, L. Angew. Chem., Int. Ed. 2011, 50, 3236.

[9] Qian, D.; Zhang, J. Chem. Commun. 2011, 47, 11152.

[10] He, W.; Li, C.; Zhang, L. J. Am. Chem. Soc. 2011, 133, 8482.

[11] Qian, D.; Zhang, J. Chem. Commun. 2012, 48, 7082.

[12] He, W.; Xie, L.; Xu, Y.; Xiang, J.; Zhang, L. Org. Biomol. Chem. 2012, 10, 3168.

[13] Xu, M.; Ren, T.-T.; Li, C.-Y. Org. Lett. 2012, 14, 4902.

[14] Fu, J.; Shang, H.; Wang, Z.; Chang, L.; Shao, W.; Yang, Z.; Tang, Y. Angew. Chem., Int. Ed. 2013, 52, 4198.

[15] Xie, L.; Liang, Z.; Yan, D.; He, W.; Xiang, J. Synlett 2013, 1809. 
[16] Li, G.; Zhang, L. Angew. Chem., Int. Ed. 2007, 46, 5156.

[17] Shapiro, N. D.; Toste, F. D. J. Am. Chem. Soc. 2007, 129, 4160

[18] Li, C.-W.; Pati, K.; Lin, G.-Y.; Sohel, S. M. A.; Hung, H.-H.; Liu, R.-S. Angew. Chem., Int. Ed. 2010, 49, 9891.

[19] Yeom, H.-S.; Lee, J.-E.; Shin, S. Angew. Chem., Int. Ed. 2008, 47, 7040.

[20] Mukherjee, A.; Dateer, R. B.; Chaudhuri, R.; Bhunia, S.; Karad, S. N.; Liu, R.-S. J. Am. Chem. Soc. 2011, 133, 15372.

[21] Xiao, J.; Li, X. Angew. Chem., Int. Ed. 2011, 50, 7226.

[22] Green, T. W.; Wuts, P. G. M. Protective Groups in Organic Synthesis, Wiley, New York, 1991.

[23] Holshouser, M. H.; Kolb, M. J. Pharm. Sci. 1986, 75, 619.

[24] Rather, J. B.; Reid, E. E. J. Am. Chem. Soc. 1919, 41, 75.

[25] Paulissen, R.; Reimlinger, H.; Hayez, E.; Hubert, A. J.; Teyssié, P. Tetrahedron Lett. 1973, 14, 2233.

[26] Shinada, T.; Kawakami, T.; Sakai, H.; Takada, I.; Ohfune, Y. Tetrahedron Lett. 1998, 39, 3757.
[27] Rubottom, G. M.; Grube, J. M.; Kincaid, K. Synth. Commun. 1976, $6,59$.

[28] Demir, A. S.; Camkerten, N.; Akgun, H.; Tanyeli, C.; Mahasneh, A. S.; Watt, D. S. Synth. Commun. 1990, 20, 2279.

[29] Lee, J. C.; Jin, Y. S.; Choi, J.-H. Chem. Commun. 2001, 956.

[30] Mo, D.-L.; Dai, L.-X.; Hou, X.-L. Tetrahedron Lett. 2009, 50, 5578.

[31] Sheng, J.; Li, X.; Tang, M.; Gao, B.; Huang, G. Synthesis 2007, 1165.

[32] Ochiai, M.; Takeuchi, Y.; Katayama, T.; Sueda, T.; Miyamoto, K. K. J. Am. Chem. Soc. 2005, 127, 12244.

[33] Deng, G.; Luo, J. Tetrahedron 2013, 69, 5937.

[34] Kaila, N.; Janz, K.; De Bernardo, S.; Bedard, P. W.; Camphausen, R. T.; Tam, S.; Tsao, D. H. H.; Keith, J. C.; Nickerson-Nutter, C.; Shilling, A.; Young-Sciame, R.; Wang, Q. J. Med. Chem. 2006, 50, 21.

(Li, L.; Lu, Z.) 